\title{
MicroRNA gga-miR-200a-3p modulates immune response via MAPK signaling pathway in chicken afflicted with necrotic enteritis
}

\author{
Thu Thao Pham ${ }^{1,4+}$, Jihye Ban ${ }^{1 \dagger}$, Yeojin Hong ${ }^{1}$, Jiae Lee ${ }^{1}$, Thi Hao Vu ${ }^{1}$, Anh Duc Truong ${ }^{1,2}$, Hyun S. Lillehoj ${ }^{3}$ \\ and Yeong Ho Hong ${ }^{1 *}$ (D)
}

\begin{abstract}
MicroRNAs (miRNAs) are small non-coding RNAs that contribute to host immune response as post-transcriptional regulation. The current study investigated the biological role of the chicken (Gallus gallus) microRNA-200a-3p (ggamiR-200a-3p), using 2 necrotic enteritis (NE) afflicted genetically disparate chicken lines, 6.3 and 7.2, as well as the mechanisms underlying the fundamental signaling pathways in chicken. The expression of gga-miR-200a-3p in the intestinal mucosal layer of NE-induced chickens, was found to be upregulated during NE infection in the diseasesusceptible chicken line 7.2. To validate the target genes, we performed an overexpression analysis of gga-miR200a-3p using chemically synthesized oligonucleotides identical to gga-miR-200a-3p, reporter gene analysis including luciferase reporter assay, and a dual fluorescence reporter assay in cultured HD1 1 chicken macrophage cell lines. Gga-miR-200a-3p was observed to be a direct transcriptional repressor of ZAK, MAP2K4, and TGF 32 that are involved in mitogen-activated protein kinase (MAPK) pathway by targeting the $3^{\prime}$-UTR of their transcripts. Besides, gga-miR200a-3p may indirectly affect the expression of protein kinases including $p 38$ and ERK1/2 at both transcriptional and translational levels, suggesting that this miRNA may function as an important regulator of the MAPK signaling path-

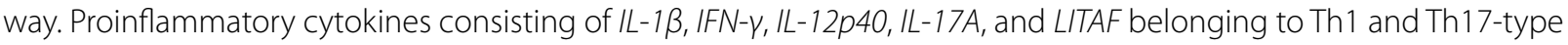
cytokines, were upregulated upon gga-miR-200a-3p overexpression. These findings have enhanced our knowledge of the immune function of gga-miR-200a-3p mediating the chicken immune response via regulation of the MAPK signaling pathway and indicate that this miRNA may serve as an important biomarker of diseases in domestic animals.
\end{abstract}

\section{Introduction}

Necrotic enteritis (NE), a disease which occurs primarily in avian species, is caused by high levels of Clostridium perfringens (C.P) [1]. C.P is a gram-positive, spore-forming anaerobe typically found in low abundance $\left(<10^{4} \mathrm{cfu}\right)$ in the gastrointestinal tract (GIT) of most bird species [2]. However, excessive C.P counts, particularly in the small intestine, lead to the onset of NE [3, 4]. C.P produce

\footnotetext{
*Correspondence: yhong@cau.ac.kr

Thu Thao Pham and Jihye Ban contributed equally to this work.

${ }^{1}$ Department of Animal Science and Technology, Chung-Ang University, Anseong 17546, Republic of Korea

Full list of author information is available at the end of the article
}

toxins and the intestinal mucosa may be covered with a fibrino-necrotic layer [5]. Such gut epithelial damage is frequently associated with coccidiosis caused by the coccidian genus, Eimeria [6]. NE outbreaks commonly occur in 17-18 days old broiler chickens [7]. Affected birds show symptoms such as huddling, ruffled feathers, inappetence, lowered growth rates, feed conversion efficacy, and diarrhea, which leads to high mortality rates $[5,8$, 9]. At first, the use of antimicrobials such as antibiotic growth promoters (AGPs) and other therapeutic agents effectively reduced NE, and they were used worldwide. However, in response to emerging concerns regarding antimicrobial resistance, the use of antimicrobials in 
poultry production has been banned from 2006 in the EU and from 2012 in Korea [10]. Thus, effective new methods capable of controlling NE, which causes serious economic loss and affects animal welfare, are needed. In addition, research investigating immunological and pathological avian host response to C.P and Eimeria maxima (E.M) has been limited.

Several functional studies on miR-200a have been conducted in vertebrates. In human and mice, it was reported that MALAT1 affected proliferation, migration, invasion, and apoptosis during the progression of hypoxic hepatocellular carcinoma by sponging miR-200a [11]. Moreover, miR-200a mediated the proliferation of hepatic stellate cells and development of fibrosis by targeting the $3^{\prime}$-UTR of SIRT1 via the SIRT1/Notch signal pathway [12]. It was also involved in protecting thymosin $\beta-4$ in cardiac microvascular endothelial cells following hypoxia/reoxygenation injury via the NRF2 antioxidant pathway [13]. Moreover, expression of miR-200a was downregulated in fibrostenosing Crohn's disease [14], HBV-induced hepatocellular carcinoma [15] and human glioma [16], thereby highlighting its function as a suppressor of many diseases. In chicken, gga-miR-200a regulated cell differentiation and proliferation of breast muscle by target 3'-UTR of GRB2 [17]. Additionally, gga-miR-200-3p was expressed in high abundance between 14 weeks and 22 weeks, and it also targeted TGFB3 related to TGFbeta signaling pathway and MAPK signaling pathway in abdominal adipose tissue during postnasal late development [18]. In response against Reticuloendotheliosis Virus, gga-miR-200a-3p was negatively correlated with CTLA4, a negative regulator of $\mathrm{T}$ cell activation in spleen [19]. Interestingly, gga-miR-200a-3p was found to be significantly up-regulated in the susceptible line $7.2 \mathrm{com}$ pared to the resistant line 6.3 in response to Necrotic Enteritis in our previous study [20]. However, the regulatory mechanism of gga-miR-200a-3p in the immune response has remained uninvestigated.

The current study analyzed the biological role of ggamiR-200a-3p as a regulator of chicken immune response and investigated its target genes, with a specific focus on those involved in the MAPK signaling pathway. We focused on gaining a better understanding of miR-200a expression following NE-induction in 2 highly inbred chicken lines and validated the target genes of gga-miR200a-3p using a chicken macrophage cell line, HD11.

\section{Materials and methods}

\section{Necrotic enteritis disease model animals}

Two White Leghorn chicken lines, line 6.3 and line 7.2, which have been highly inbred and maintained since 1931, show resistance or susceptibility to avian leucosis virus (ALV) and Marek's disease virus (MDV), respectively [21]. To induce NE, the chickens were challenged with E.M strain $41 \mathrm{~A}\left(1.0 \times 10^{4}\right.$ oocysts/birds $)$ by oral gavage at day 14 after hatching, followed by challenge with C.P strain Del $\left(1.0 \times 10^{9} \mathrm{cfu} / \mathrm{bird}\right)$ by oral gavage for the next 2 days, (day 4 following E.M infection). The infection experiment was extended for 6 days. Intestinal mucosal layers (IMLs) were collected from 5 chickens per group following NE induction. The IMLs samples were provided by the Animal Biosciences and Biotechnology Laboratory (Beltsville, MD, USA) of the United States Department of Agriculture (USDA)-Agricultural Research Service. All animal protocols were approved by the Institutional Animal Care and Use Committees of the Beltsville Agricultural Research Center (Protocol \#09019). The IMLs were carefully homogenized after freezing with liquid nitrogen, and total RNA was extracted using TRIzol (Invitrogen, Carlsbad, CA, USA).

\section{Target gene prediction of gga-miR-200a-3p}

Prediction of the target genes of gga-miR-200a-3p was carried out via miRDB v6.0 [22], which contains chicken miRNA as well as mRNA data, and provides a custom prediction mode based on mature miRNAs sequences. Genes with a target score of more than 80 were further functionally analyzed using the DAVID Bioinformatic Resources [23, 24] and KEGG PATHWAY Database [25], leading to the mapping of genes involved in immunerelated pathways, such as the MAPK signaling pathway, TGF-beta pathway and/or toll-like receptor signaling pathway. Next, potential targets were predicted based on their meeting these criteria and on the basis of the presence of gga-miR-200a-3p binding sites in their $3^{\prime}$-UTR [26].

\section{Cell culture, mimic miRNA and LPS stimulation}

The HD11 chicken macrophage cell line [27] was cultured in RPMI-1640 medium, supplemented with L-glutamine (Gibco, Grand Island, NY, USA), 10\% heat-inactivated fetal bovine serum (Gibco) and 1\% penicillin-streptomycin (Gibco). The cells were incubated under humidified conditions in a $5 \% \mathrm{CO}_{2}$ atmosphere at $41{ }^{\circ} \mathrm{C}$.

Based on the precursor sequence of gga-miR-200a-3p (MI0001249) obtained from miRbase catalogs [28], an oligonucleotide gga-miR-200a-3p mimic was chemically synthesized by Bioneer (Daejeon, Republic of Korea) for overexpression of the miRNA in cells. The experimental group consisted of 4 treatments as follows: (1) control; (2) exposure to lipopolysaccharide (LPS) of Salmonella enteritis (S.E); (3) mimic miR-200a overexpression; and (4) mimic miR-200a overexpression upon exposure to LPS.Each group consisted of 3 independent replicates. To overexpress the mimic miRNA, synthetic mimic gga-miR-200a-3p oligonucleotides were transiently 
transfected into the HD11 chicken macrophage cell line seeded in a 12-well plate at a final concentration of $50 \mathrm{nM}$ using Lipofectamine 3000 (Invitrogen, Carlsbad, CA, USA) diluted in Opti-MEM medium (Gibco), according to the manufacturer's instructions. Following $20 \mathrm{~h}$ of transient transfection, either LPS $(1.0 \mu \mathrm{g} / \mathrm{mL})$ (Sigma-Aldrich, St. Louis, MO, USA) or normal medium were used to stimulate the treated cells for the $4 \mathrm{~h}$. The control group was treated with similar amounts of Lipofectamine 3000 (Invitrogen) and Opti-MEM (Gibco) without the oligonucleotides. Samples were collected from each group for further experimentation.

\section{Construction of gga-miR-200a-3p or candidate target gene 3'-UTR constructs}

The oligonucleotide sequence of mature gga-miR200a-3p was cloned into a pDsRed2-N1 plasmid, containing a cytomegalovirus (CMV) promoter carrying the red fluorescence protein (RFP) (Clontech, Palo Alto, CA, USA), using NotI digestion ( $5^{\prime}$ and $\left.3^{\prime}\right)$ (Additional file 1 ). This construct, named miR200a/DsRed simultaneously expressed a small fragment of miRNA molecule and RFP for visualizing the RNA molecule. Regions of the 3 '-UTR flanking the predicted miR-200a binding sites in the candidate target genes were amplified using pooled cDNA of ADOL chicken lines as templates; the specific forward and reverse primers used have been provided (Additional file 1). For the dual fluorescence assay, the partial $3^{\prime}$-UTR of TGF $\beta 2$ was ligated into a pcDNA3 plasmid, containing enhanced green fluorescence protein (eGFP) using NotI/ XbaI restriction enzymes. The resulting construct was called TGF $\beta 2 / E G F P$. For the luciferase assay, the target genes PCR products were digested via SacI/HindIII and cloned into a pMIR-REPORT Luciferase vector (Ambion, Austin, TX, USA), resulting in "Gene"/Luc recombinant constructs independently containing $T G F \beta 2, Z A K$ and $M A P 2 K 4$. The ligated constructs were transformed into top 10 competent Escherichia coli (E. coli) (Invitrogen) and recombinant plasmids were confirmed by sequencing (Genotech, Daejeon, Republic of Korea).

\section{Quantitative real-time PCR (qRT-PCR) for mRNA and miRNA}

Total RNA from chicken lines 6.3 and 7.2, and HD11 cells was isolated using Trizol reagent (Invitrogen) in accordance with the manufacturer's instructions. cDNAs from mRNA were synthesized using a RevertAid First Strand cDNA Synthesis kit (Thermo Scientific, Waltham, MA, USA). In brief, the reaction mixture $(20 \mu \mathrm{L})$ contained RNA $(2 \mu \mathrm{g}), 5 \times$ Reaction Buffer $(4 \mu \mathrm{L}), 10 \mathrm{mM}$ dNTP Mix $(2 \mu \mathrm{L})$, Oligo $(\mathrm{dT})_{18}$ primers $(1 \mu \mathrm{L})$, RiboLock RNase Inhibitor (1 U), and RevertAid M-MuLV RT (10 U). The reaction was subjected to the following conditions: $60 \mathrm{~min}$ at $42{ }^{\circ} \mathrm{C}$, followed by $70{ }^{\circ} \mathrm{C}$ for $5 \mathrm{~min}$. For miRNA
cDNA synthesis, a Ncode ${ }^{\mathrm{TM}}$ miRNA First-strand cDNA synthesis Kit (Invitrogen) was used as per the manufacturer's protocol. Diluted amounts of cDNA were used as templates to perform quantitative RT-PCR (qRT-PCR) as per manufacturer's instructions. Specific forward primers for miRNAs were designed and a universal primer was used as the reverse primer. Primers for mRNAs were designed using Primer-BLAST [29] (Additional file 1). qRT-PCR was performed using $2 \times$ Power SYBR Green Master Mix (Roche Life Science, Mannheim, Germany) and the LightCycler ${ }^{\circledR} 96$ System (Roche Life Science) in accordance with the manufacturer's instructions. Threshold cycle $(\mathrm{Ct})$ values were normalized to those of GAPDH (mRNA) or U1A small nuclear RNA (miRNA) by the $2^{-\Delta \Delta \mathrm{Ct}}$ method [30].

\section{Western blotting}

The HD11 chicken macrophage cells transfected with gga-miR-200a-3p mimic or gga-miR-200a-3p mimic in the background of endotoxins LPS stimulation were analyzed for protein expression. Protein samples, the concentrations of which were measured by a BCA assay, were electrophoresed on SDS-PAGE gels containing 10\% polyacrylamide and transferred onto polyvinylidene difluoride (PVDF) membranes (GE Healthcare, Rydalmere, Australia). The PVDF membranes were blocked with $5 \%$ non-fat milk containing $0.05 \%$ Tween 20 in PBS (pH 7.4) (PBST) for $1 \mathrm{~h}$. Primary antibodies including rabbit anti-chicken phosphor-p44/42 MAPK (Cell Signaling Technology, ERK1/2; Thr202/Tyr204, \#4370, specific), rabbit anti-chicken phospho-p38 MAPK (Cell Signaling Technology, Thr180/Tyr182, \#4631, specific), and mouse anti-chicken GAPDH antibodies (Thermo Fisher Scientific, AM4300, specific) were prepared based on the specific dilution ratio of each primary antibody in PBST containing $2 \%$ non-fat milk for overnight incubation with the PVDF membranes at $4{ }^{\circ} \mathrm{C}$. After washing with PBST, membranes were treated with horseradish peroxidase (HRP)-linked anti-rabbit or anti-mouse (Thermo Fisher Scientific) secondary antibodies (based on the primary antibodies) diluted in PBST containing 2\% non-fat milk and incubated for $2 \mathrm{~h}$ at room temperature. Band signals were detected using Western Lightning ECL Plus substrate (Thermo Fisher Scientific) and were exposed on Hyperfilm (GE Healthcare).

\section{Dual fluorescence assay}

The HD11 macrophage cells were seeded at $1.0 \times 10^{6}$ cells/well and cultured in 12-well plates (Corning, NY, USA). When cells reached $80 \%$ confluency, the cells were transfected with miR200a/DsRed or TGF $\beta 2 / E G F P$, or cotransfected with both plasmids using Lipofectamine 3000 (Invitrogen) as per the manufacturer's instructions. Three 
hours after transfection, $50 \mu \mathrm{M}$ of $\beta$-mercaptoethanol was added to each well. Following $48 \mathrm{~h}$ of transfection, eGFP or DsRed were visualized using an EVOS $^{\circledR}$ FL Color Imaging System (Life Technologies). The settings of the fluorescence microscope were as follows: $470 \mathrm{~nm}$ excitation filter and $525 \mathrm{~nm}$ emission filter for green fluorescent protein; and $530 \mathrm{~nm}$ excitation filter and $593 \mathrm{~nm}$ emission filter for red fluorescent protein.

\section{Luciferase reporter assay}

The HD11 macrophage cells were seeded at $1.0 \times 10^{6}$ cells/well and cultured in 12-well plates (Corning). "Gene"/Luc were co-transfected along with miR200a/ DsRed into the cells using Lipofectamine 3000 (Invitrogen) as per the manufacturer's instructions; the pMIRREPORT $\beta$-gal control plasmid (Ambion) was also transfected to normalize transfection efficiency. The cells were collected after $24 \mathrm{~h}$ and lysed by $1 \times$ luciferase cell culture lysis reagent (Promega). After centrifugation at $20000 \times g$ for $1 \mathrm{~min}$, the supernatant was used for the measurement of the luciferase and $\beta$-galactosidase activity in 96-well plates (Corning) using Luciferase Assay Systems (Promega, Madison, WI, USA) and $\beta$-galactose solution (o-nitrophenyl- $\beta$-D-galactopyranoside or OPNG), respectively, as per the manufacturer's instructions. $\beta$-galactosidase activities were used to normalize the reported luciferase activities. All experiments were independently replicated thrice to verify the results.

\section{Statistical analysis}

All in vitro experiments were performed in triplicate, and differences between groups were analyzed using oneway ANOVA followed by Duncan's multiple range test using IBM SPSS software (SPSS 25.0 for Windows; IBM, Chicago, IL, USA). Data for each group $(N=3)$ were expressed as mean \pm standard error of mean (SEM). Statistical significance was set at $p<0.05$.

\section{Results}

Analysis of gga-miR-200a-3p structure and candidate gene predictions

Gga-miR-200a-3p seed sequences among 6 species, human, monkey, mouse, cow, chicken and lizard were completely identical, indicating that these were highly conserved across vertebrates. In chicken, gga-miR-200a$3 p$ was located between $g g a-m i R-429$ and $g g a-m i R-200 b$, on chromosome 21 (Figure 1). The miRDB algorithm, which was used for miRNA target gene prediction, revealed a total of 555 candidate target genes of gga-miR$200 \mathrm{a}-3 \mathrm{p}$, primarily based on the sequence of gga-miR200a-3p. Next, the genes were sorted based on target score, whereby 102 candidate target genes were found to display scores of over 90 (Additional file 2). Among them, four genes were involved in immune-related pathways, as predicted by DAVID and KEGG tools, among them, three were involved in the MAPK signaling pathway. These three genes, sterile alpha motif and leucine zipper

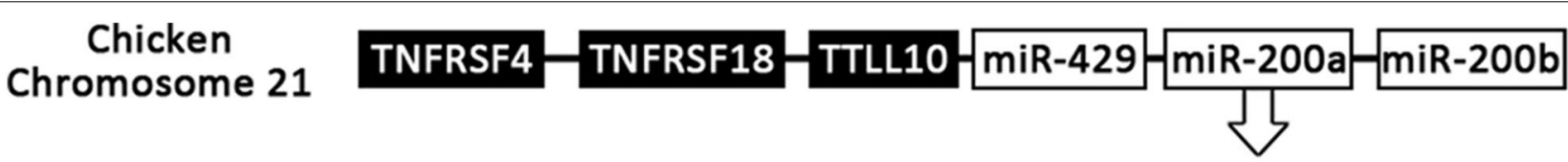 \\ hsa-mir-200a \\ mml-mir-200a \\ mu-mir-200a \\ bta-mir-200a \\ gga-mir-200a \\ aca-mir-200a \\ GGGCCCCUGUGAGCAUCUUACCGGACAGUGCUGGAUUUCCCAGCUUGACUCUAACACUGUCUGGUAACGAUGUUCAAAGG. UGAC GGGCCCCUGUGAGCAUCUUACCGGACAGUGCUGGAUUUCCCAGCUUGACUCUAACACUGUCUGGUAACGAUGUUCAAAGG. UGAC GGGCCUCUGUGGGCAUCUUACCGGACAGUGCUGGAUUUCUUGGCUUGACUCUAACA CUGUCUGGUAACGAUGUUCAAAGG. UGAC GGGCCUCUGUGGACAUCUUACCGGACAGUGCUGGAUUUCUCGGCUCGACUCUAACACUGUCUGGUAACGAUGUUCAAAGG . UGAC GGUCCUCUGUGGGCAUCUUACUAGACAGUGCUGGAUUUCUUGGAUCUAUUCUAACACUGUCUGGUAACGAUGUUUAAAGGGUGAA GGUCCUCUGUGGACAUCUUACUAGACAGUGCUGGAUUGUGUGCUCUGAUUCUAACACUGUCUGGUAACGAUGUUCAAAGGGUGAG

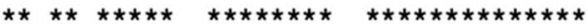

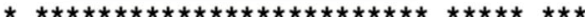 \\ 5' UAACACUGUCUGGUAACGAUGU 3' Seed Sequence \\ Stem-loop structure of pre-gga-miR-200a \\ Mature sequence of gga-miR-200a-3p \\ Figure 1 Chromosomal position of chicken miR-200a and comparison of miR-200a sequences among several species. Chicken miR-200a is located between miR-429 and miR-200b on chromosome 21. MiR-200a-3p is highly conserved across many species (hsa, Homo sapiens; mml, Macaca mulatta; mmu, Mus musculus; bta, Bos taurus; gga, Gallus gallus; Aca, Anolis carolinensis). The mature miR-200a-3p sequences of each species are in underlined in blue font and seed sequences are in underlined red font.}


containing kinase $(Z A K)$; transforming growth factor beta 2 (TGFB2); and mitogen-activated protein kinase kinase 4 (MAP2K4), were selected for further experimental validation. The binding sites between $3^{\prime}$-UTR of each candidate and gga-miR-200a-3p were predicted by the RNA-hybrid software tool, based on seed matches between the two molecules (nucleotides 2-8 of the miRNA and 3'-UTR of candidate genes) (Figure 2).

\section{Gga-miR-200a-3p and the expression of its target genes in two highly inbred chicken lines}

To determine the expression of gga-miR-200a-3p and putative target genes associated with NE-induction, qRTPCR was carried using CDNA of IML samples from NEafflicted and control groups (both MD-resistant line 6.3 and MD-susceptible line 7.2). On day 6 following NEinduction, the expression of gga-miR-200a-3p in IML was significantly increased in line 7.2 , but not in line 6.3 (Figure 3A). Furthermore, the expression of all three candidate target genes was significantly downregulated only in line 7.2 (Figure 3B). These results indicate that expression of each candidate target gene was negatively correlated with that of gga-miR-200a-3p in line 7.2.

\section{Changes in target gene expression upon treatment with synthetic chicken miR-200a and LPS in chicken macrophage cell line}

In order to investigate whether the expression of candidate target genes can be altered by overexpressing ggamiR-200a-3p upon LPS stimulation-an endotoxins stimulus, we transfected gga-miR-200a-3p mimic into the HD11, and treated the cells with LPS from S.E. The expression of gga-miR-200a-3p in mimic miRNA transfected cells alone and in cells treated with LPS following mimic miRNA transfection, significantly increased compared to that of the control, indicating that gga-miR200a-3p was successfully induced (Figure 4A). Among the three candidate target genes, the expression of $Z A K$ mRNA decreased significantly in cells transfected with mimic miRNA alone (Figure $4 \mathrm{C}$ ), while the expression of $T G F \beta 2, Z A K$ and $M A P 2 K 4$ mRNA in cells treated with LPS following mimic miRNA transfection was strongly decreased $(p<0.05)$ relative to that in cells stimulated using only LPS (Figures 4B-D). However, the expression of gga-miR-200a-3p and its target gene $Z A K$ displayed no changes in response to LPS from S.E compared to the control (Figures 4A, C), whereas significant increases were observed in the expression of TGF $\beta 2$ and MAP $2 K 4$ (fivefold and 14-fold, respectively) upon LPS treatment (Figures 4B, D). These results indicate that overexpression of gga-miR-200a-3p may down-regulate the expression of TGFB2, ZAK, and MAP $2 K 4$.

\section{In vitro target gene validation of chicken miR-200a-3p in HD11 chicken macrophage cell line}

To identify whether miR-200a represses candidate target genes by targeting their predicted seed region in $3^{\prime}$-UTR site, a luciferase reporter assay and a dual fluorescence reporter assay were performed in HD11 cells.Each target gene site complementary to gga-miR-200a-3p was predicted using the RNA-hybrid computational analysis pro-

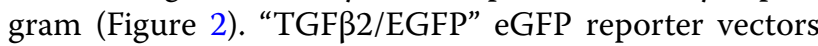
were constructed (Figure 5A). Following transfection of miR200a/DsRed and/or TGF $32 / E G F P$ vector, eGFP and DsRed protein expression were analyzed by fluorescence microscopy. In cells co-transfected with both miR200a/ DsRed and TGF $32 /$ EGFP constructs, DsRed and GFP fluorescence was significantly reduced compared to that

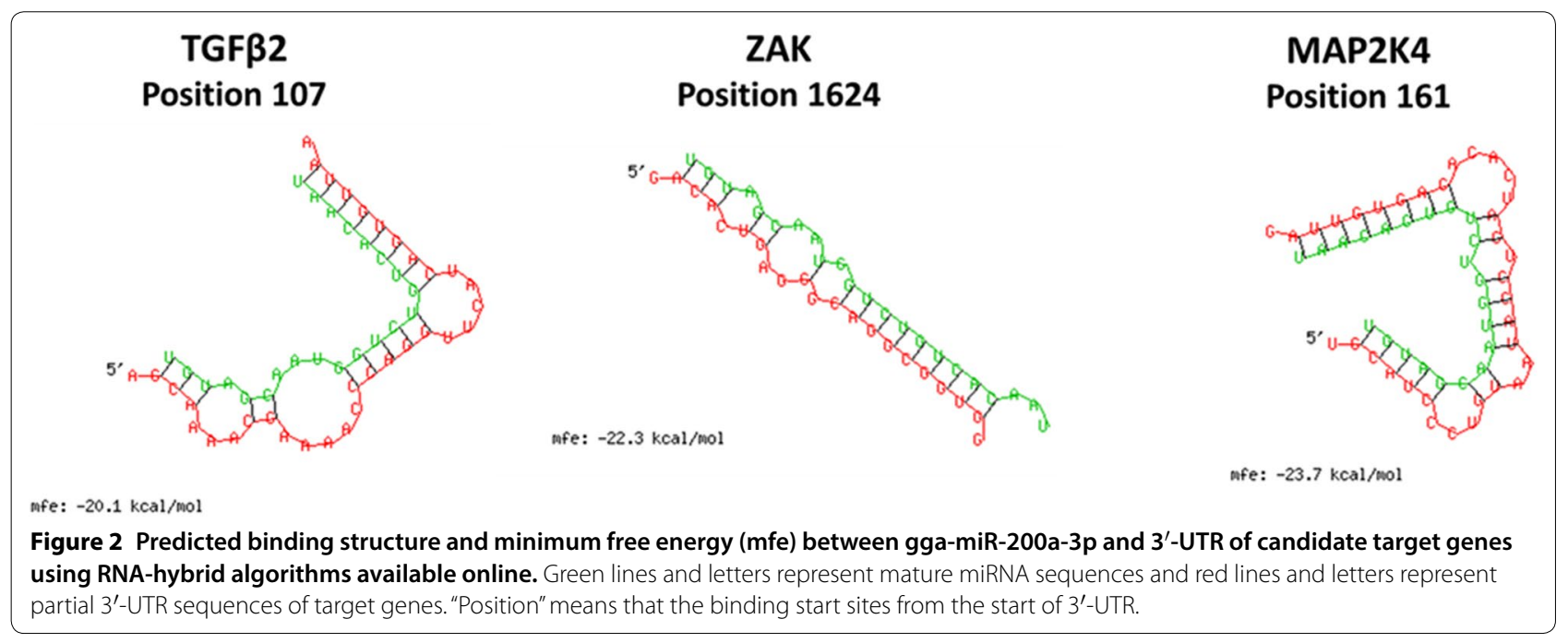




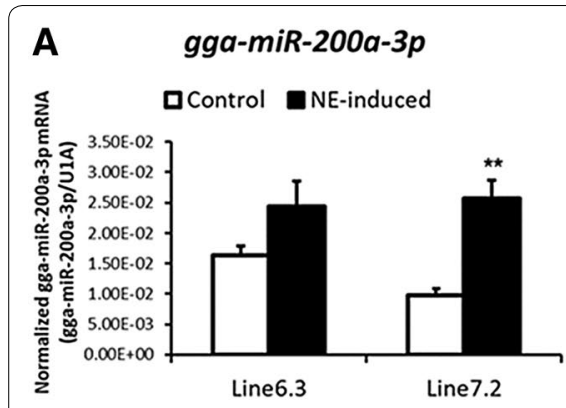

B

TGF $\beta 2$

ZAK

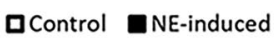

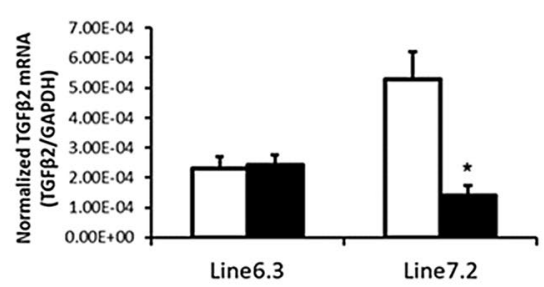

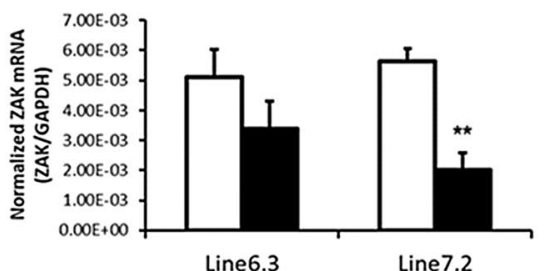

MAP2K4

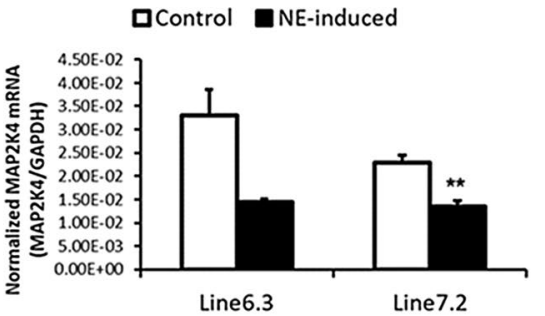

Figure 3 The expression profile of gga-miR-200a-3p a and its candidate target genes $\mathbf{b}$ in IML derived from line 6.3 and line 7.2 . The expression levels of genes and miRNA were normalized to that of the glyceraldehyde-3-phosphate dehydrogenase (GAPDH) and U1A, respectively. Significant differences in gene expression levels between control and treatment (NE-induced) were analyzed by the Student's $t$ test and indicated as follows: ${ }^{*}=P<0.05$ and ${ }^{* *}=P<0.01$. Error bars indicate the SEM of technical replicates that were done in triplicate.
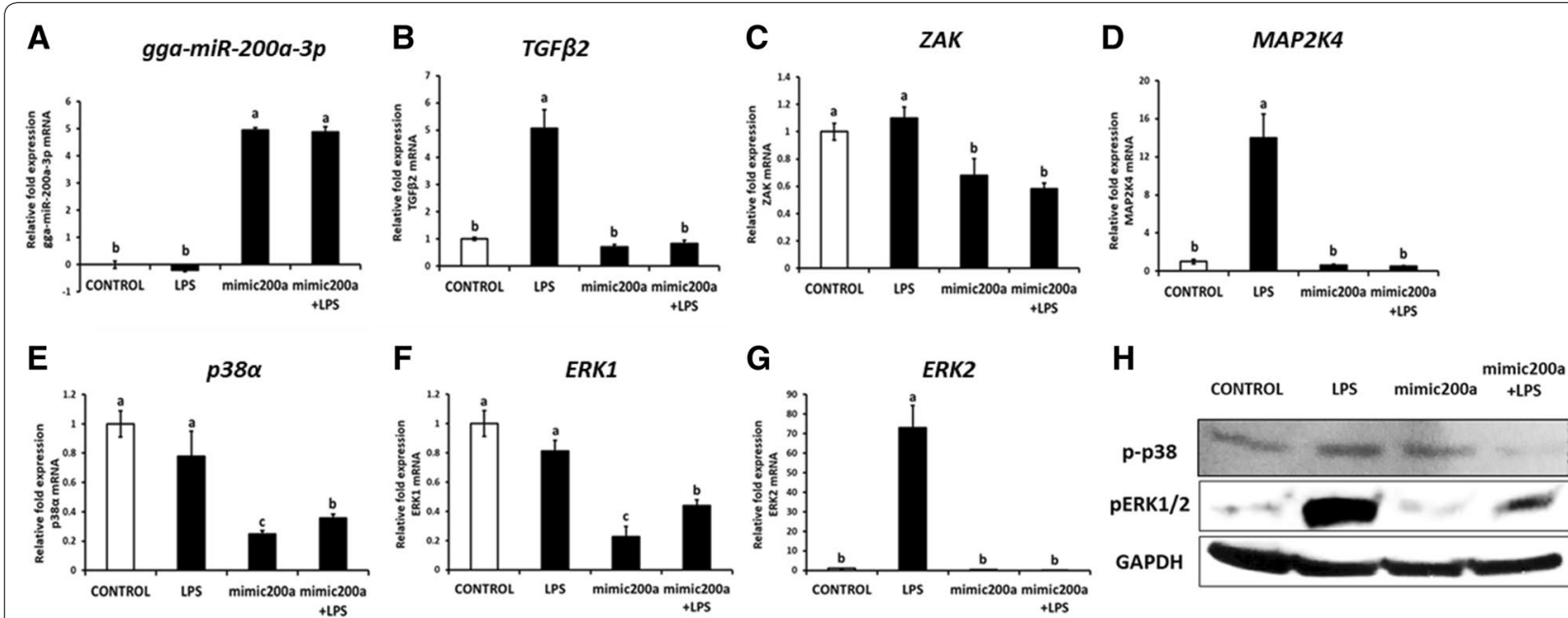

Figure 4 Gga-miR-200a-3p repressed the expression of the immune target genes related to MAPK signaling pathway. The expression profile of gga-miR-200a-3p A its candidate target genes. B-D The signaling molecules of MAPK signaling pathway $\mathbf{E}-\mathbf{G}$ and Western blot analysis of the signaling molecules $\mathbf{H}$ in the HD11 chicken macrophage cell line treated with mimic miRNA and LPS.Expression data were transformed to a relative fold expression value in arbitrary units (AU) using the control expression level as a calibrator. Results are expressed as the mean \pm SEM $(n=3)$ of three independent experiment.

in the control (Figure 5B). These results indicate that gga-miR-200a-3p might repress $T G F \beta 2$ by targeting its 3'-UTR.

Structures of the luciferase reporter vectors including TGF 2 2, MAP2K4, and $Z A K$ were constructed. In addition, the DsRed reporter vector constructed as named miR200a/DsRed (Figure 5A). The miR200a/ DsRed construct was co-transfected into the HD11 cell line along with a "Gene"/Luc vector or an empty luciferase vector as a control. Target gene expression 

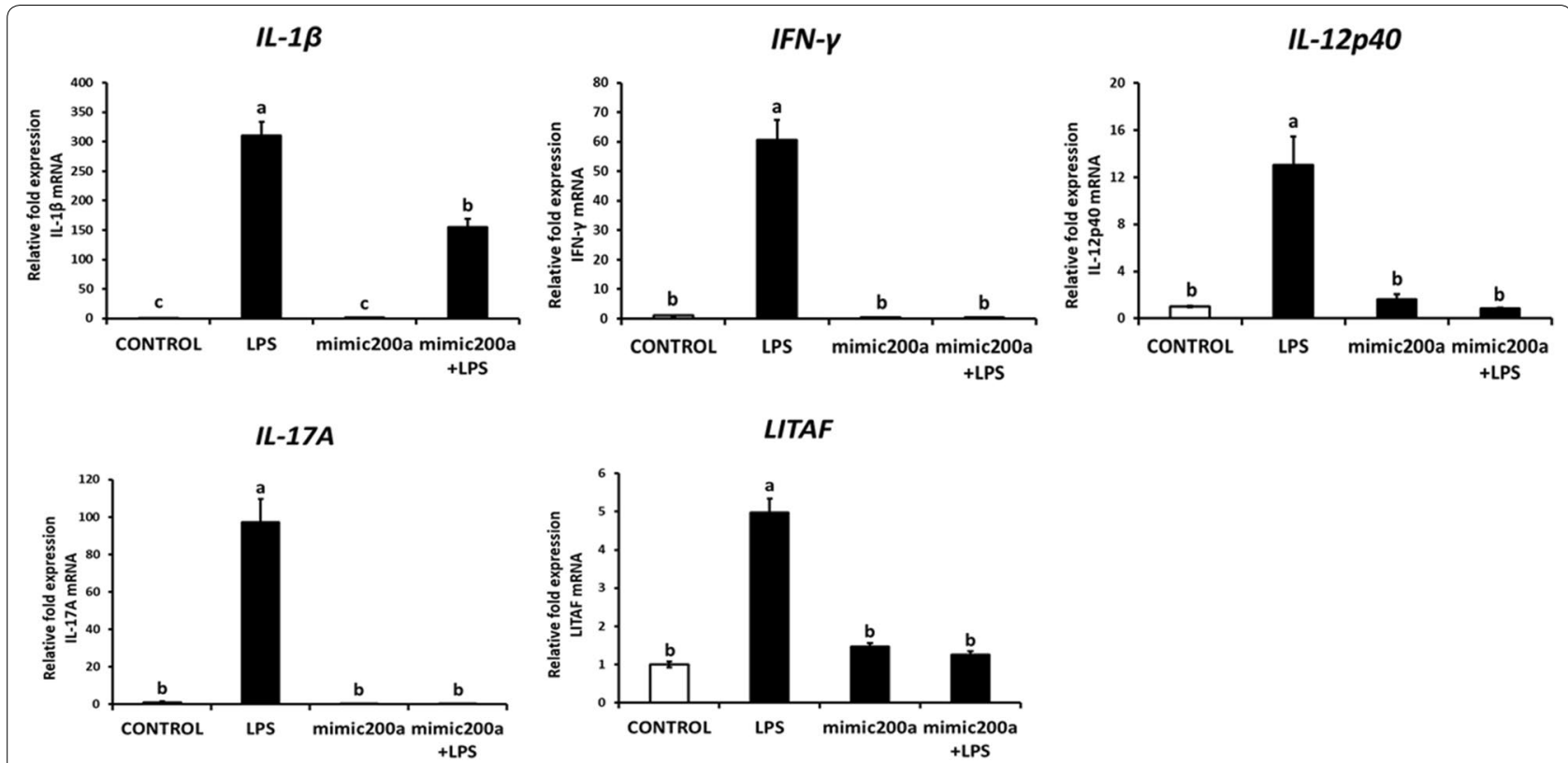

Figure 5 Expression profile of cytokine genes involved in the MAPK signaling pathway of the HD11 chicken macrophage cell line treated with mimic miRNA and LPS. Data are expressed as each mRNA level normalized against the GAPDH mRNA level. Data are expressed as the mean \pm SEM $(n=3)$ of three independent experiments.

patterns indicated that luciferase activity was significantly decreased when miR200a/DsRed was co-transfected with TGF $\beta 2 / \mathrm{Luc}$, ZAK/Luc or MAP2K4/Luc, compared with their control groups (Figure 5C). These results suggest that gga-miR-200a-3p may directly target $T G F \beta 2, M A P 2 K 4$, and $Z A K$.

\section{Gga-miR-200a-3p inhibits the MAPK signaling pathway}

Extracellular signal-regulated protein kinase (ERK) and the p38 MAP kinase are the two major MAP kinases that significantly contribute to innate immune response [31]. The levels of $p 38 \alpha$ and ERK1 mRNA in mimic-miR-200a transfected groups were downregulated by fivefold compared to that in the control. While no differences were observed between mimicmiR-200a-transfected samples and the control with respect to ERK2 expression (Figures $4 \mathrm{E}-\mathrm{H}$ ). However, upon LPS treatment following transfection with mimic-miR-200a, the mRNA levels of $E R K 1, p 38 \alpha$ were downregulated by twofold, and ERK2 by 70 -fold (Figures $4 \mathrm{E}-\mathrm{G}$ ). Further, we also analyzed the protein levels of p38 and ERK1/2 via Western blotting; the results showed that mimic-miR-200a inhibited the expression of p-p38 and pERK1/2 in the background of LPS treatment (Figure $4 \mathrm{H}$ ). The downstream components of the MAPK signaling pathway, pro-inflammatory cytokines, including $I L-1 \beta, I F N-\gamma$, and $I L-12 p 40$ which are Th1 type cytokines; $I L-17 A$ which is a member of the Th17 cytokine family and the LITAF-proinflammatory mediator were significantly downregulated in the mimic-miR-200a transfected LPS-stimulated group compared with that in the only LPS treated group (Figure 6). Especially, the mRNA expression of $I F N-\gamma$ and $I L-17 A$ which was remarkably repressed upon mimicmiR-200a overexpression, was strongly upregulated by approximately 60 -fold and 100-fold, respectively, upon LPS treatment (Figure 6). These results indicate that mimic-200a may negatively regulate the MAP kinaseassociated pathway and suppress the expression of proinflammatory cytokines in the HD11 chicken macrophage cell line.

\section{Discussion}

An increasing number of studies are being conducted on the crucial role played by microRNAs, such as ggamiR-1306-5p [32], gga-miR-10a [33], gga-miR-130b-3p [34], in mediating the innate host response of chickens against pathogens. The current study investigated the biological function of gga-miR-200a-3p in the immune response. Analysis of C.P- and E.M-induced NE disease infections showed that miR-200a expression increased in chicken line 7.2, which was susceptible to ALV and MDV. By contrast, the expression of genes predicted to be gga-miR-200a-3p targets, and which were involved in the MAPK pathway showed an inverse correlation to 

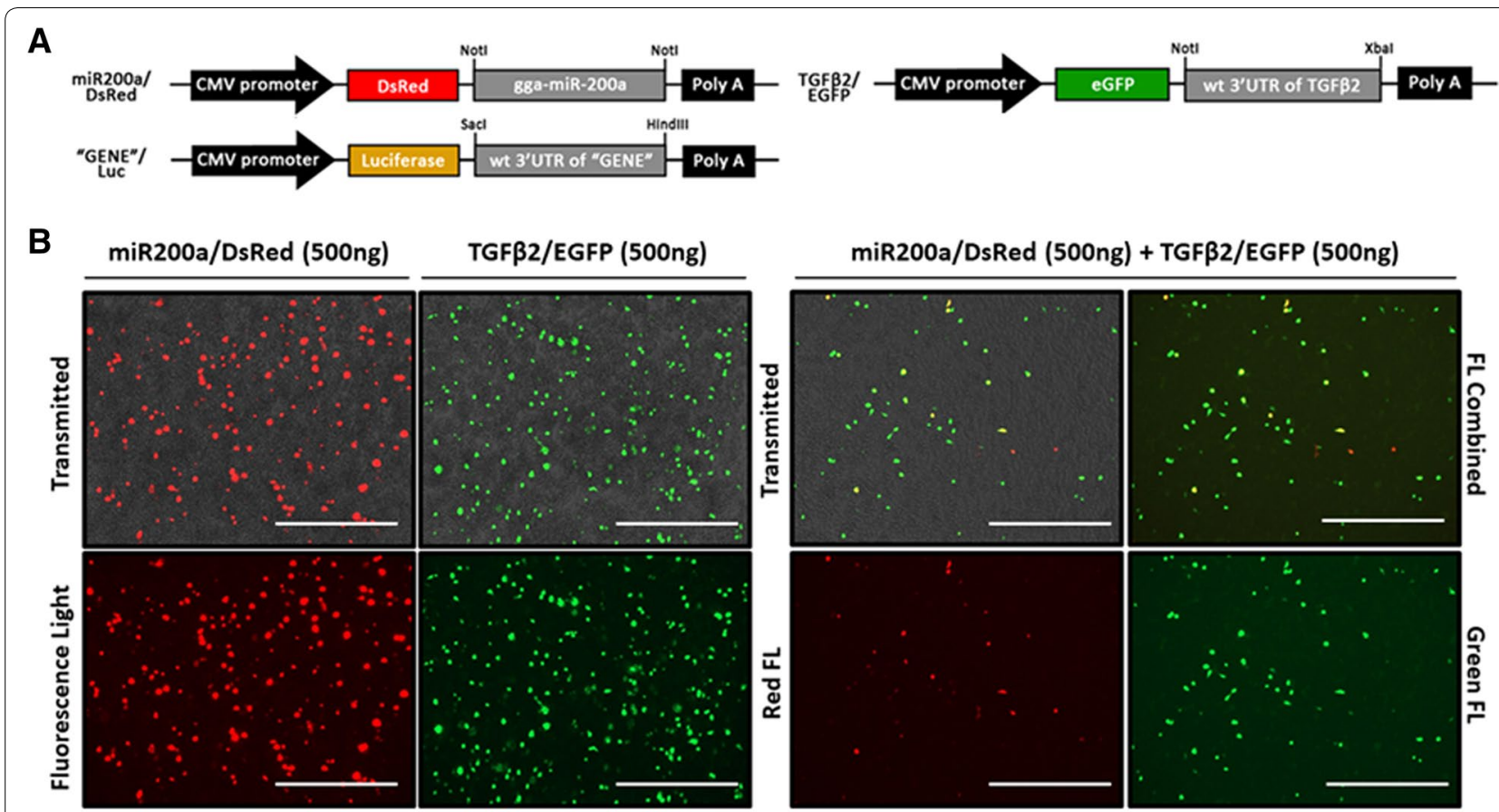

C
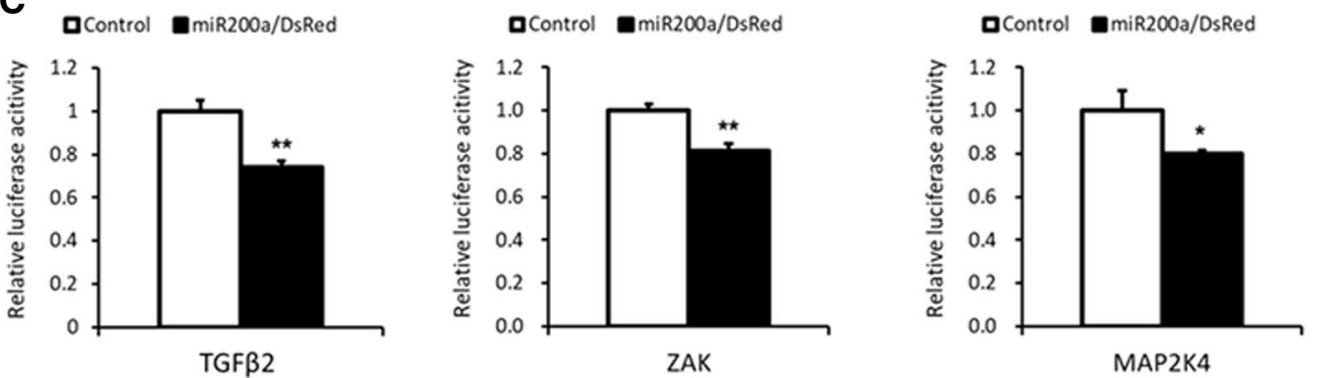

Figure 6 Constructed vectors for this study and in vitro target validation of gga-miR-200a-3p. A Schematic diagrams of expression vector for DsRed with gga-miR-200a-3p, eGFP with partial 3'-UTR of TGFB2 and Luciferase with partial 3'-UTR of selected genes. The miR200a/DsRed vector expresses both red fluorescence protein (RFP) and gga-miR-200a-3p; TGF $32 / E G F P$ vector expresses both enhanced green fluorescence protein (eGFP) and cloned 3'-UTR of TGF $\beta 2$. The "Gene"/Luc vectors express both luciferase and cloned 3'-UTR of TGF 32 , MAP2K4, or ZAK and named as TGF $32 / L u c, M A P 2 K 4 / L u c$, and ZAK/Luc, respectively. The two restriction sites for cloning are indicated at both sides of inserted gene. B miR-200a/ DsRed and TGF $\beta 2 /$ eGFP were co-transfected into HD1 1 chicken macrophage cell line in a dual fluorescence reporter. After $48 \mathrm{~h}$ of co-transfection, eGFP and DsRed expression were examined under a fluorescence microscope. White bars indicate $400 \mu \mathrm{m}$ in length. C Luciferase reporter assay conducted for $24 \mathrm{~h}$ following co-transfection of "Gene"/Luc vectors with miR200a/DsRed or luciferase vector with miR200a/DsRed as the control in the HD11 chicken macrophage cell line. Results (mean \pm SEM) are representative of three independent experiments.

that of miR-200a in chicken line 7.2, which is also susceptible to C.P and E.M pathogens.

In vitro experiments were conducted using the chicken macrophage cell line to validate the target genes of miR200a. Similar to those from mammals, macrophages from avian species also play important roles in adaptive immunity by producing regulatory molecules such as cytokines, enzymes, and receptors. Avian macrophages also function in innate immunity by performing phagocytic and microbicidal functions [35].
Several target genes of miR-200a have been validated; these genes are associated with the immune signaling pathway including E-cadherin repressors, the wnt $\beta$ catenin signaling pathway in gastric adenocarcinoma, $Z E B 1 / Z E B 2$ [36] and suppression of castration-resistant prostate cancer by inhibiting the activation of BRD4mediated AR signaling in humans [37]. However, to the best of our knowledge, no target gene of miR-200a has been validated in chickens until now and no studies have been conducted on pathways mediated by miR-200a. The 
qRT-PCR data revealed that the overexpression of miR200a repressed the expression of MAP2K4,ZAK, and $T G F \beta 2$ mRNA. In addition, a luciferase reporter assay confirmed that chicken $M A P 2 K 4, Z A K$, and TGF 32 were targeted by gga-miR-200a-3p. The results of the co-transfection experiments using constructs encoding eGFP-3'-UTR of TGF 32 and DsRed-miR-200a revealed that miR-200a directly bound the $3^{\prime}$-UTR of TGF 2 and silenced its expression. Our identification of chicken $T G F \beta 2$ as a target of gga-miR-200a-3p is consistent with earlier reports in mice and humans [38, 39] and, to our knowledge, for the first time, two other genes, MAP2K4 and $Z A K$, were also identified as the targets of gga-miR200a-3p in this study. $Z A K$ is a MAPK-kinase kinase (MKKK) which was involved in the activation of the ERK, p38 signaling pathways $[40,41]$. In this study, LPS did not induce the expression of ZAK at the transcriptional level, but it did induce $M A P 2 K 4$ at the transcriptional level p-p38 and pERK1/2 at the translational level in the chicken macrophage cell line. Importantly, MAP2K4 has been demonstrated to be a direct activator of MAP kinases that promote human prostate cancer metastasis [42] and act as prognostic markers of osteosarcoma tumorigenesis [43]. Although LPS did not successfully induce mRNAs level of $p 38 \alpha$ and ERK1 in MAPK signaling pathway, the target genes of gga-miR-200a-3p might perform a vital function as inducers in the MAPK signaling pathway, while gga-miR-200a-3p was identified as its inhibitor.

The MAPK pathway is activated by many kinds of stimuli including endotoxic lipopolysaccharides (LPS), hyperosmolarity [44], proinflammatory cytokines and factors such as IL-1 $\beta$, and PAF [45, 46] and viral infections [47]. In the present study, activation of the MAPK signaling pathway was induced by LPS stimulation. Additionally, gga-miR-200a-3p also inhibited the activity of the MAPK signaling pathway by suppressing MAPK signaling members of families, including $p 38$ and $E R K 1 / 2$, to mediate the production of proinflammatory cytokines. Proinflammatory cytokines, $I L-1 \beta, I L-12 p 40$, and $I F N-\gamma$, were induced by Th1 type cells; Th17 type cells induce following molecules such as $I L-17 A$ and LITAF which function as an inflammatory mediator [48] that were all up-regulated upon LPS treatment in our study. These proinflammatory cytokines were triggered due to host immune response via activation of the MAPK signaling pathway [49] which was shown to be repressed by gga-miR-200a-3p.

In conclusion, we demonstrated that gga-miR200a-3p, a type of endogenous small noncoding RNA, is involved in transcriptional and translational regulation of genes related to the MAPK signaling pathway in chicken. The result indicates that susceptible line
7.2 to NE disease might be altered by the upregulation of gga-miR-200a-3p which suppressed the target genes, MAP2K4, ZAK and TGF 2 , which are involved in the MAPK signaling pathway, leading to a cascade inhibition of downstream kinase signaling molecules such as $E R K 1 / 2, p 38$ and proinflammatory cytokines in chicken macrophage cell line. These results provide an understanding of the biological functioning of ggamiR-200a-3p in the host innate immune response and mechanisms underlying the gga-miR-200a-3p-mediated MAPK signaling pathway upon NE induction in chicken.

\section{Supplementary information}

Supplementary information accompanies this paper at https://doi. org/10.1186/s13567-020-0736-x.

Additional file 1: Primer sequences used for quantitative real-time PCR and cloning.

Additional file 2: List of putative target genes of gga-200a-3p predicted by miRDB. Genes involved in immune-related pathway were identified using DAVID functional annotation tool, and KEGG pathway.

\section{Abbreviations}

MAPK signaling pathway: mitogen-activated protein kinase signaling pathway; TGFß2: transforming growth factor beta-2; MAP2K4: mitogen-activated protein kinase kinase 4; ZAK: the sterile alpha motif and leucine zipper containing kinase; ERK1/2: extracellular signal-regulated protein kinases 1 and 2; LPS: lipopolysaccharide; IL: interleukin; LITAF: lipopolysaccharide-induced tumor necrosis factor-alpha factor.

\section{Acknowledgements}

The authors thank the Avian Disease and Oncology Laboratory (ADOL, East Lansing, MI) of the USDA-Agricultural Research Service for providing the chicken lines used in this study.

\section{Authors' contributions}

The authors made the following contributions: TTP, JB, and YHH conceived and designed the experiments; $\mathrm{YHH}$ and HSL contributed reagents, materials, and analytical tools; JB, TTP, ADT, YH, JL, and THV performed the experiments; TTP, JB, and YHH analyzed and interpreted the data; and JB, TTP, and YHH wrote the manuscript. All authors read and approved the final manuscript.

\section{Funding}

This work was supported by the Next-Generation BioGreen 21 Program (No. PJ01324202 and PJ01324203), Rural Development Administration, Republic of Korea, and an NIFA grant (\#2017-6701526793) from the USDA.

\section{Availability of data and materials}

All data generated or analyzed during this study are included in this published article and its supplementary information files.

\section{Ethics approval and consent to participate}

The experiments were approved by the Beltsville Area Institutional Animal Care and Use Committee, United States, Department of Agriculture (USDA)Agricultural Research Service (Protocol \#09-019).

\section{Competing interests}

The authors declare that they have no competing interests.

\section{Author details}

${ }^{1}$ Department of Animal Science and Technology, Chung-Ang University, Anseong 17546, Republic of Korea. ${ }^{2}$ Department of Biochemistry and Immunology, National Institute of Veterinary Research, 86 Truong Chinh, Dong Da, 
Hanoi 100000, Viet Nam. ${ }^{3}$ Animal Biosciences and Biotechnology Laboratory, Agricultural Research Services, United States Department of Agriculture, Beltsville, MD 20705, USA. ${ }^{4}$ Key Laboratory of Animal Cell Biotechnology, National Institute of Animal Science, 9 Tan Phong, Thuy Phuong, Bac Tu Liem, Hanoi 100000, Viet Nam.

Received: 14 October 2019 Accepted: 27 December 2019 Published online: 03 February 2020

\section{References}

1. McDevitt RM, Brooker JD, Acamovic T, Sparks NHC (2006) Necrotic enteritis: a continuing challenge for the poultry industry. Worlds Poult Sci J 62:221-247

2. Willis AT (2014) Anaerobic bacteriology clinical and laboratory practice. Butterworth-Heinemann, Oxford

3. Asaoka Y, Yanai T, Hirayama H, Une Y, Saito E, Sakai H, Goryo M, Fukush H, Masegi T (2004) Fatal necrotic enteritis associated with Clostridium perfringens in wild crows (Corvus macrorhynchos). Avian Pathol 33:19-24

4. Myers GSA, Rasko DA, Cheung JK, Ravel J, Seshadri R, DeBoy RT, Ren Q, Varga J, Awad MM, Brinkac LM (2006) Skewed genomic variability in strains of the toxigenic bacterial pathogen, Clostridium perfringens. Genome Res 16:1031-1040

5. Porter RE Jr (1998) Bacterial enteritides of poultry. Poult Sci 77:1159-1165

6. Williams RB (2005) Intercurrent coccidiosis and necrotic enteritis of chickens: rational, integrated disease management by maintenance of gut integrity. Avian Pathol 34:159-180

7. Tech R (1999) Necrotic enteritis and associated conditions in broiler chickens. World Poult 15:44-47

8. Kaldhusdal M, Hofshagen M (1992) Barley inclusion and avoparcin supplementation in broiler diets. 2. Clinical, pathological, and bacteriological findings in a mild form of necrotic enteritis. Poult Sci 71:1145-1153

9. Lovland A, Kaldhusdal M (2001) Severely impaired production performance in broiler flocks with high incidence of Clostridium perfringensassociated hepatitis. Avian Pathol 30:73-81

10. Oakley BB, Talundzic E, Morales CA, Hiett KL, Siragusa GR, Volozhantsev NV, Seal BS (2011) Comparative genomics of four closely related Clostridium perfringens bacteriophages reveals variable evolution among core genes with therapeutic potential. BMC Genomics 12:282

11. Zhao Z-B, Chen F, Bai X-F (2019) Long Noncoding RNA MALAT1 regulates hepatocellular carcinoma growth under hypoxia via sponging MicroRNA200a. Yonsei Med J 60:727-734

12. Yang J-J, Tao H, Liu L-P, Hu W, Deng Z-Y, Li J (2017) miR-200a controls hepatic stellate cell activation and fibrosis via SIRT1/Notch1 signal pathway. Inflamm Res 66:341-352

13. Li Y, Zhu X, Liu X, Du A, Yu B (2017) Mir-200a mediates protection of thymosin $\beta-4$ in cardiac microvascular endothelial cells as a novel mechanism under hypoxia-reoxygenation injury. J Cell Biochem 120:19098-19106

14. Mehta SJ, Lewis A, Nijhuis A, Jeffery R, Biancheri P, Di Sabatino A, Feakins R, Silver A, Lindsay JO (2018) Epithelial down-regulation of the miR-200 family in fibrostenosing Crohn's disease is associated with features of epithelial to mesenchymal transition. J Cell Mol Med 22:5617-5628

15. Shi T, Hua Q, Ma Z, Lv Q (2017) Downregulation of miR-200a-3p induced by hepatitis $B$ Virus $X(\mathrm{HBX})$ protein promotes cell proliferation and invasion in HBV-infection-associated hepatocarcinoma. Pathol Pract 213:1464-1469

16. Liu Y, Chen M-B, Cheng L, Zhang Z, Yu Z, Jiang Q, Chen G, Cao C (2018) MicroRNA-200a downregulation in human glioma leads to Gai1 overexpression, Akt activation, and cell proliferation. Oncogene 37:2890-2902

17. Jiang K, Zhang M, Li F, Li D, Sun G, Liu X, Li H, Han R, Jiang R, Li Z (2017) Study on the role of gga-miRNA-200a in regulating cell differentiation and proliferation of chicken breast muscle by targeting Grb2. Animal Cells Syst (Seoul) 21:365-373

18. Chen Y, Zhao Y, Jin W, Li Y, Zhang Y, Ma X, Sun G, Han R, Tian Y, Li H (2019) MicroRNAs and their regulatory networks in Chinese Gushi chicken abdominal adipose tissue during postnatal late development. BMC Genomics 20:778

19. Gao S, Jiang H, Sun J, Diao Y, Tang Y, Hu J (2019) Integrated analysis of miRNA and mRNA expression profiles in spleen of specific pathogen-free chicken infected with avian reticuloendotheliosis virus strain SNV. Int J Mol Sci 20:E1041

20. Dinh H, Hong YH, Lillehoj HS (2014) Modulation of microRNAs in two genetically disparate chicken lines showing different necrotic enteritis disease susceptibility. Vet Immunol Immunopathol 159:74-82

21. Bacon LD, Hunt HD, Cheng HH (2000) A review of the development of chicken lines to resolve genes determining resistance to diseases. Poult Sci 79:1082-1093

22. Wong N, Wang X (2014) MiRDB: an online resource for microRNA target prediction and functional annotations. Nucleic Acids Res 43:D146-D152

23. Huang DW, Sherman BT, Lempicki RA (2009) Systematic and integrative analysis of large gene lists using DAVID bioinformatics resources. Nat Protoc 4:44

24. Huang DW, Sherman BT, Lempicki RA (2008) Bioinformatics enrichment tools: paths toward the comprehensive functional analysis of large gene lists. Nucleic Acids Res 37:1-13

25. Kanehisa M, Goto S (2000) KEGG: kyoto encyclopedia of genes and genomes. Nucleic Acids Res 28:27-30

26. Rehmsmeier M, Steffen P, Höchsmann M, Giegerich R (2004) Fast and effective prediction of microRNA/target duplexes. RNA 10:1507-1517

27. Beug H, von Kirchbach A, Döderlein G, Conscience J-F, Graf T (1979) Chicken hematopoietic cells transformed by seven strains of defective avian leukemia viruses display three distinct phenotypes of differentiation. Cell 18:375-390

28. Kozomara A, Birgaoanu M, Griffiths-Jones S (2018) MiRBase: from microRNA sequences to function. Nucleic Acids Res 47:D155-D162

29. Ye J, Coulouris G, Zaretskaya I, Cutcutache I, Rozen S, Madden TL (2012) Primer-BLAST: a tool to design target-specific primers for polymerase chain reaction. BMC Bioinformatics 13:134

30. Livak KJ, Schmittgen TD (2001) Analysis of relative gene expression data using real-time quantitative PCR and the $2^{-\triangle \Delta C T}$ method. Methods 25:402-408

31. Dong C, Davis RJ, Flavell RA (2002) MAP kinases in the immune response. Annu Rev Immunol 20:55-72

32. Sun W, Liu R, Li P, Li Q, Cui H, Zheng M, Wen J, Zhao G (2019) Chicken ggamiR-1306-5p targets Tollip and plays an important role in host response against Salmonella enteritidis infection. J Anim Sci Biotechnol 10:59

33. Pham TT, Ban J, Lee K, Hong Y, Lee J, Truong AD, Lillehoj HS, Hong YH (2019) MicroRNA gga-miR-10a-mediated transcriptional regulation of the immune genes in necrotic enteritis afflicted chickens. Dev Comp Immunol 102:103472

34. Yuan B, Zou M, Zhao Y, Zhang K, Sun Y, Peng X (2018) Up-regulation of miR-130b-3p activates the PTEN/PI3K/AKT/NF-KB pathway to defense against Mycoplasma gallisepticum (HS strain) infection of chicken. Int J Mol Sci 19:E2172

35. Qureshi MA, Heggen CL, Hussain I (2000) Avian macrophage: effector functions in health and disease. Dev Comp Immunol 24:103-119

36. Cong N, Du P, Zhang A, Shen F, Su J, Pu P, Wang T, Zjang J, Kang C, Zhang Q (2013) Downregulated microRNA-200a promotes EMT and tumor growth through the wnt/ $\beta$-catenin pathway by targeting the E-cadherin repressors ZEB1/ZEB2 in gastric adenocarcinoma. Oncol Rep 29:1579-1587

37. Guan H, You Z, Wang C, Fang F, Peng R, Mao L, Xu B, Chen M (2019) MicroRNA-200a suppresses prostate cancer progression through BRD4/ AR signaling pathway. Cancer Med 8:1474-1485

38. Lu R, Ji Z, Li X, Qin J, Cui G, Chen J, Zhai Q, Zhao C, Zhang W, Yu Z (2015) Tumor suppressive microRNA-200a inhibits renal cell carcinoma development by directly targeting TGFB2. Tumor Biol 36:6691-6700

39. Wang B, Koh P, Winbanks C, Coughlan MT, McClelland A, Watson A, Jandeleit-Dahm K, Burns WC, Thomas MC, Cooper ME (2011) miR-200a prevents renal fibrogenesis through repression of TGF- $\beta 2$ expression. Diabetes 60:280-287

40. Liu T-C, Huang C-J, Chu Y-C, Wei C-C, Chou C-C, Chou M-Y, Chou C-K, Yang $\mathrm{J}-\mathrm{J}$ (2000) Cloning and expression of ZAK, a mixed lineage kinase-like protein containing a leucine-zipper and a sterile-alpha motif. Biochem Biophys Res Commun 274:811-816

41. Gotoh I, Adachi M, Nishida E (2001) Identification and characterization of a novel MAP kinase kinase kinase, MLTK. J Biol Chem 276:4276-4286

42. Pavese JM, Ogden IM, Voll EA, Huang X, Xu L, Jovanovic B, Bergan RC (2014) Mitogen-activated protein kinase kinase 4 (MAP2K4) promotes human prostate cancer metastasis. PLoS One 9:e102289 
43. Tesser-Gamba F, Petrilli AS, de Seixas Alves MT, Garcia Filho RJ, Juliano Y, Toledo SRC (2012) MAPK7 and MAP2K4 as prognostic markers in osteosarcoma. Hum Pathol 43:994-1002

44. Han J, Lee JD, Bibbs L, Ulevitch RJ (1994) A MAP kinase targeted by endotoxin and hyperosmolarity in mammalian cells. Science 265:808-811

45. Kitanaka N, Nakano R, Sugiura K, Kitanaka T, Namba S, Konno T, Nakayama T, Sugiya H (2019) Interleukin-1 $\beta$ promotes interleulin-6 expression via ERK1/2 signaling pathway in canine dermal fibroblasts. PLoS One 14:e0220262

46. Jun S, Lee $\mathrm{S}$, Kim H-C, Ng C, Schneider AM, Ji H, Ying H, Wang H, DePinho RA, Park J-I (2013) PAF-mediated MAPK signaling hyperactivation via LAMTOR3 induces pancreatic tumorigenesis. Cell Rep 5:314-322
47. DuShane JK, Maginnis MS (2019) Human DNA Virus Exploitation of the MAPK-ERK Cascade. Int J Mol Sci 20:3427

48. Bettelli E, Oukka M, Kuchroo VK (2007) TH-17 cells in the circle of immunity and autoimmunity. Nat Immunol 8:345-350

49. Hommes DW, Peppelenbosch MP, Van Deventer SJH (2003) Mitogen activated protein (MAP) kinase signal transduction pathways and novel anti-inflammatory targets. Gut 52:144-151

\section{Publisher's Note}

Springer Nature remains neutral with regard to jurisdictional claims in published maps and institutional affiliations.
Ready to submit your research? Choose BMC and benefit from:

- fast, convenient online submission

- thorough peer review by experienced researchers in your field

- rapid publication on acceptance

- support for research data, including large and complex data types

- gold Open Access which fosters wider collaboration and increased citations

- maximum visibility for your research: over 100M website views per year

At BMC, research is always in progress.

Learn more biomedcentral.com/submissions 